\title{
Blood pressure influences end-stage renal disease of Cd151 knockout mice
}

\author{
Norman Sachs, ${ }^{1}$ Nike Claessen, ${ }^{2}$ Jan Aten, ${ }^{2}$ Maaike Kreft, ${ }^{1}$ Gwendoline J.D. Teske, ${ }^{2}$ \\ Anneke Koeman, ${ }^{3}$ Coert J. Zuurbier, ${ }^{3}$ Hans Janssen, ${ }^{1}$ and Arnoud Sonnenberg ${ }^{1}$
}

${ }^{1}$ Division of Cell Biology, The Netherlands Cancer Institute, Amsterdam, The Netherlands. 2Department of Pathology and ${ }^{3}$ Department of Anesthesiology, Academic Medical Center (AMC), University of Amsterdam, Amsterdam, The Netherlands.

\begin{abstract}
Podocytes of the kidney adhere tightly to the underlying glomerular basement membrane (GBM) in order to maintain a functional filtration barrier. The clinical importance of podocyte binding to the GBM via an integrin-laminin-actin axis has been illustrated in models with altered function of $\alpha 3 \beta 1$ integrin, integrinlinked kinase, laminin-521, and $\alpha$-actinin 4 . Here we expanded on the podocyte-GBM binding model by showing that the main podocyte adhesion receptor, integrin $\alpha 3 \beta 1$, interacts with the tetraspanin CD151 in situ in humans. Deletion of Cd151 in mouse glomerular epithelial cells led to reduced adhesive strength to laminin by redistributing $\alpha 3 \beta 1$ at the cell-matrix interface. Moreover, in vivo podocyte-specific deletion of $C d 151$ led to glomerular nephropathy. Although global Cd151-null B6 mice were not susceptible to renal disease, as has been shown previously, increasing blood and transcapillary filtration pressure induced nephropathy in these mice. Importantly, blocking the angiotensin-converting enzyme in renal disease-susceptible global Cd151-null FVB mice prolonged their median life span. Together, these results establish CD151 as a crucial modifier of integrin-mediated adhesion of podocytes to the GBM and show that blood pressure is an important factor in the initiation and progression of Cd151 knockout-induced nephropathy.
\end{abstract}

\section{Introduction}

The human kidney produces approximately $180 \mathrm{l}$ of filtrate per day, forced through the glomerular filtration barrier along a transcapillary pressure gradient. Podocytes, which line the outer aspects of the glomerular basement membrane (GBM), are therefore exposed to considerable mechanical stress. In order to maintain a functional filtration barrier, podocytes must counteract (pathological) pressure gradients by adhering strongly to the GBM (1). Podocyte adhesion is primarily mediated by the extracellular engagement of heterodimeric integrin $\alpha 3 \beta 1$ to the GBM component laminin-521. Subsequent intracellular coupling to the actin cytoskeleton provides mechanical reinforcement. The importance of an intact GBM-integrin-actin axis is illustrated by glomerular diseases in mice with podocyte-specific genetic ablation of Itga3 (2), $\operatorname{Itgb1}(3,4)$, and integrin-linked kinase $(5,6)$ as well as Pierson syndrome and focal segmental glomerulosclerosis in humans, which are caused by mutations in laminin-521 and $\alpha$-actinin 4, respectively $(7,8)$. In contrast, the contribution of dystroglycan to podocyte adhesion seems minor, as podocyte-specific Dag1knockout mice do not display glomerular pathology or aggravate the renal phenotype of Itga3-null animals (9). Mice deficient for the tetraspanin Cd151 develop kidney abnormalities similar to, albeit less severe than, those of mice with podocyte-specific Itga3 knockout $(2,10)$. Importantly, only mice bred onto certain genetic backgrounds (e.g., FVB, 129P2/FVB) exhibit renal pathology in the absence of Cd151, whereas other strains (e.g., 129Sv, C57BL/6) are resistant, indicative of the presence of genetic modifiers $(2,10,11)$. Functionally, Cd151 has been suggested to alter $\alpha 3 \beta 1$-mediated adhesion and/or to influence maturation of the $\operatorname{GBM}(2,10)$. In humans, a rare frameshift mutation in CD151 causes hereditary nephritis in association with localized skin blistering, sensorineu-

Conflict of interest: The authors have declared that no conflict of interest exists. Citation for this article: J Clin Invest. 2012;122(1):348-358. doi:10.1172/JCI58878. ral deafness, and $\beta$-thalassemia minor (12). Subcellularly, CD151 associates with the integrins $\alpha 3$ (13) and $\alpha 6$ (14), which both bind laminin and are required for epithelial integrity (15-17). The interaction with $\alpha 3 \beta 1$ is highly stoichiometric and depends on an extracellular Gln-Arg-Asp (QRD) sequence in CD151 (13). CD151 is able to increase the laminin-511/521-binding activity of $\alpha 3$ in liposome-binding assays in vitro (18). However, to our knowledge, whether podocyte CD151 forms a functional complex with $\alpha 3$ and alters integrin-mediated adhesion in vivo has not previously been established. Here, we addressed these questions and showed that kidney failure caused by the absence of Cd151 can be ameliorated by decreasing mechanical stress imposed on podocytes.

\section{Results}

CD151 and $\alpha 3$ bind at the basal site of human podocytes in vivo. The interaction between CD151 and $\alpha 3$ is well established in vitro, and both proteins are strongly expressed in podocytes $(13,15,19)$. To investigate whether renal CD151 binds $\alpha 3$ in vivo, cryosections of healthy human kidneys were subjected to immunofluorescence analysis. CD151 strongly colocalized with $\alpha 3$ in the glomerular epithelium (Figure 1A). Using an in situ proximity ligation assay (PLA), we verified that the 2 proteins interacted in podocytes (Figure $1 \mathrm{~B})$. In fetal human kidneys, we found that CD151- $\alpha 3$ complex formation increased after the early capillary loop stage of developing glomeruli, which correlated with increased expression of the 2 molecules (Supplemental Figure 1; supplemental material available online with this article; doi:10.1172/JCI58878DS1). Using immunogold transmission electron microscopy, we found CD151 to be distinctly enriched at the basal site of podocyte foot processes that contact the GBM (Figure 1C).

Podocyte-specific CD151 knockout mice develop kidney abnormalities. To further elucidate the role of podocyte CD151, we generated mice with podocyte-specific conditional knockout of Cd151 (referred to herein as $C d 151^{f l f l}$ mice; Supplemental Figure 2) and 
A

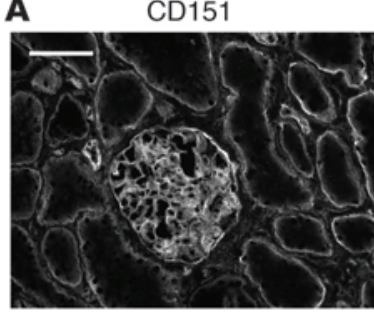

$\alpha 3$

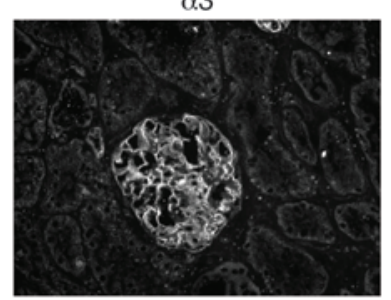

CD151/a3

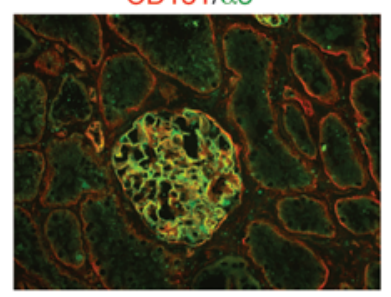

B
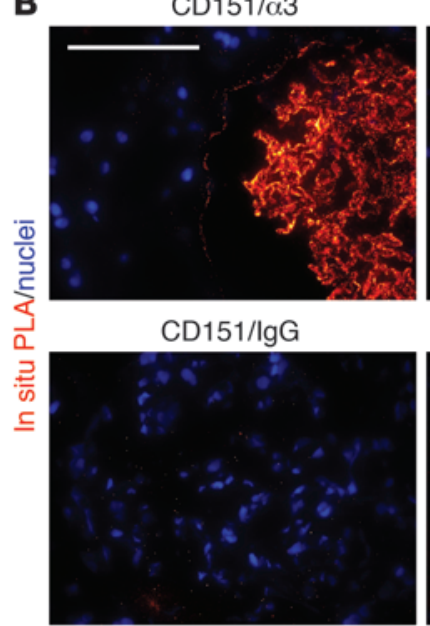

C

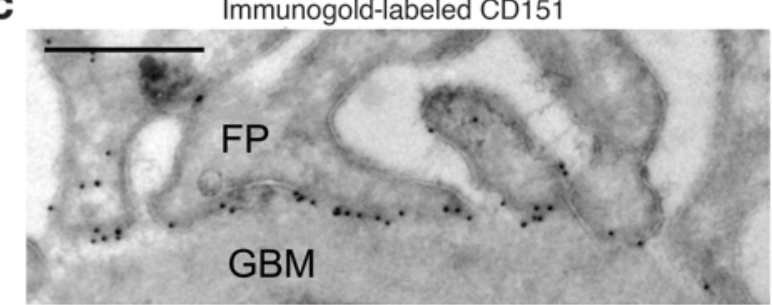

\section{Figure 1}

CD151 binds to $\alpha 3$ at the cell-matrix interaction site of human podocytes in vivo. (A) Colocalization of CD151 and integrin $\alpha 3$ in a glomerulus of a human kidney cryosection, shown by immunofluorescence. (B) Strongly positive in situ PLA of CD151 and $\alpha 3$ in a human glomerulus along with positive $(\alpha 3 / \beta 1)$ and negative controls ( $\alpha 3$ and CD151 with appropriate control IgGs). (C) Transmission electron micrograph showing immunogold-labeled CD151 enriched at the basal membrane of podocyte foot processes (FP) in contact with the GBM. Scale bars: $50 \mu \mathrm{m}(\mathbf{A}$ and B), $500 \mathrm{~nm}$ (C). crossed them with mice expressing the Cre recombinase under control of the human podocin promoter (2.5P-Cre mice; ref. 20), generating $C d 151^{f l / f l} ; 2.5 \mathrm{P}-\mathrm{Cr} \mathrm{e}^{+}$and $C d 151^{f l / f l} ; 2.5 \mathrm{P}-\mathrm{Cre}^{-}$mice on an FVB (N6) background. Urine analysis of 4- to 14-week old mice by SDS-PAGE and albumin ELISA revealed albuminuria only in the Cd151 $1^{f / f l} ; 2.5 \mathrm{P}-\mathrm{Cre} \mathrm{e}^{+}$mice (Figure 2A), indicative of a dysfunctional filtration barrier. As shown in Figure 2B, we regularly observed ultrastructural defects in Cd151-deficient podocytes (abnormally broad foot processes) as well as in the adjacent GBM (focally thick and irregular) of 7-week-old animals. These conditions were similar to those previously observed in $\mathrm{Cd} 151^{-/-}$mice (2). The morphological kidney abnormalities in Cd151/l/fl $; 2.5 \mathrm{P}-\mathrm{Cre}^{+}$mice progressively worsened: whereas 5 -week-old mice displayed GBM irregularities in few glomeruli, at 23 weeks of age, most glomeruli were affected, and first proteinaceous casts were present. At 11-12 months of age, glomerulosclerosis and dilated tubuli containing proteinaceous casts were present throughout the entire kidney cortex, and mice had to be sacrificed due to excessive loss of body weight and/or peritoneal edema. In contrast, $\mathrm{Cd} 151^{f l / f l} ; 2.5 \mathrm{P}-\mathrm{Cre}{ }^{-}$littermates were without pathological findings for up to 16 months. (Supplemental Figure 3 and Figure 2C). Cell-matrix anomalies in $C d 151^{f l f f} ; 2.5 \mathrm{P}-\mathrm{Cr} \mathrm{e}^{+}$mice were confirmed by the altered distribution of nidogen and podocin (Figure 2D). Loss of podocytes by detachment seemingly did not precede the development of glo-

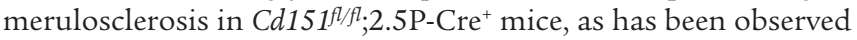
elsewhere $(1,21)$. However, podocyte numbers were significantly decreased in older $C d 151^{f l / f l} ; 2.5 \mathrm{P}-\mathrm{Cre}^{+}$mice, coinciding with the appearance of sclerosed glomeruli (Supplemental Figure 4).

Another CD151-binding integrin that has previously been suggested to maintain the filtration barrier is $\alpha 6 \beta 4(14,22)$. However, podocyte-specific deletion of Itgb4 did not cause kidney defects in mice (Supplemental Figure 5). We conclude that the kidney abnor- malities observed in $\mathrm{Cd} 151^{-/-} \mathrm{FVB}$ mice were mainly caused by the absence of $C d 151$ in podocytes. We furthermore noted that lack of $\alpha 6 \beta 4$ in podocytes (unlike $\alpha 3 \beta 1$ ) did not result in kidney failure.

CD151 strengthens $\alpha 3$-mediated adhesion of glomerular epithelial cells in vitro. Cd151-positive glomerular epithelial cells (GECs) were isolated from a Cd151fl/fl; $\operatorname{Tr} p 53^{+/-}$mouse by Dynabead perfusion (23), and floxed Cd151 alleles were recombined to generate the Cd151-deficient GEC line (Figure 3, A-D). Expression of CD151 in Cd151-negative GECs was rescued with human wild-type CD151 and human CD151 in which the integrin-binding sequence QRD at position 194-196 was mutated to INF (Figure 3E and ref. 24). Cell surface expression of the integrin subunits $\alpha 2, \alpha 3, \alpha 6, \beta 1$, and $\beta 4$ was equal in all cell lines (Supplemental Table 1 ). To elucidate how CD151 regulates adhesion at a cellular level, we performed spreading and short-term adhesion assays. Neither the percentage nor the area of spread cells on a laminin-332-rich matrix differed significantly between Cd151-positive and -negative GECs (Figure 4A). Additionally, the number of cells adhering to this matrix after 15 minutes was similar for all 4 GEC lines ( $10 \%$ of cells spread; Figure 4B). Because podocytes in vivo are fully spread and must withstand transcapillary filtration pressure, we next applied shear stress to GECs with or without Cd151 spread on a laminin-332rich matrix and quantified cellular detachment. Cd151-negative GECs showed a substantially reduced tolerance to shear stress compared with Cd151-positive GECs. The human wild-type rescue construct, but not the mutated integrin-binding sequence, conferred shear stress tolerance to Cd151-negative GECs similar to that observed for Cd151-positive GECs (Figure 4C). Thus, we concluded that adhesion strengthening depends on the integrinbinding function of CD151. As shown in Figure 4D, the adhesion benefit of CD151 on GECs was matrix specific, because no difference was observed in $50 \%$ detachment $\left(\tau_{50}\right)$ of $C d 151$-positive ver- 
A
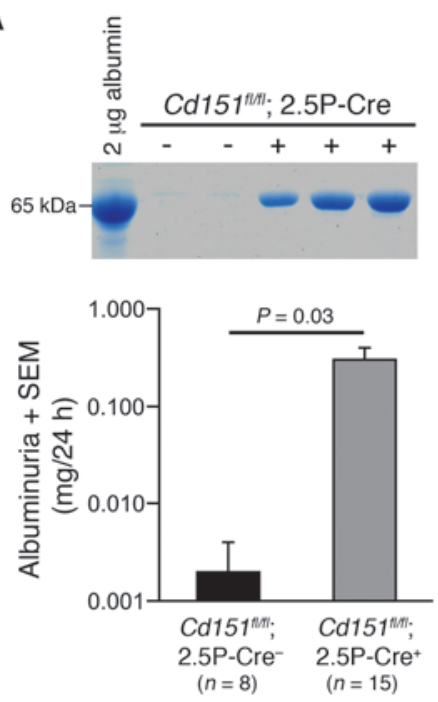

B

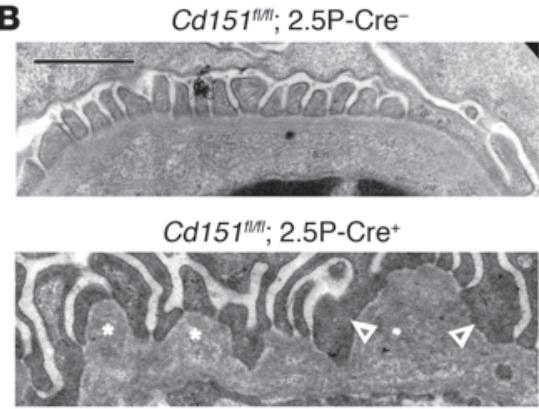

\section{C}
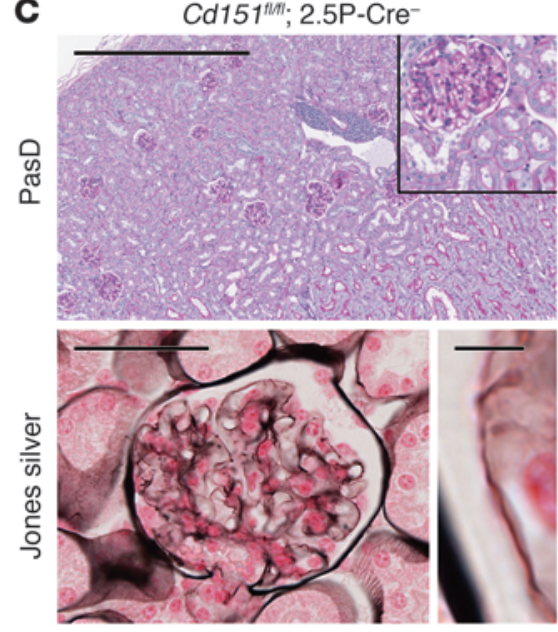

D

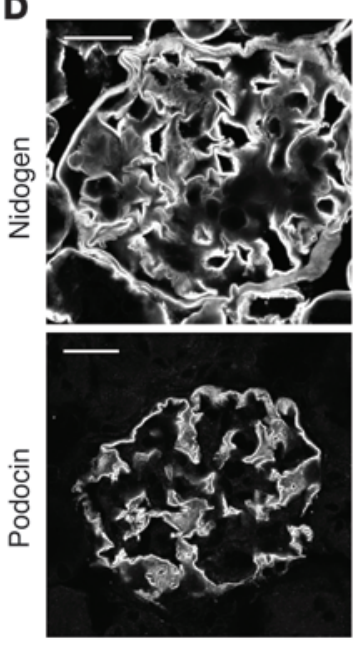

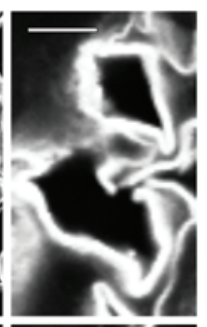

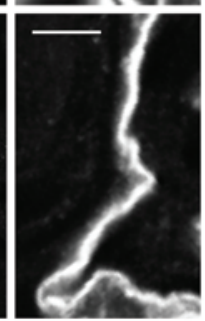

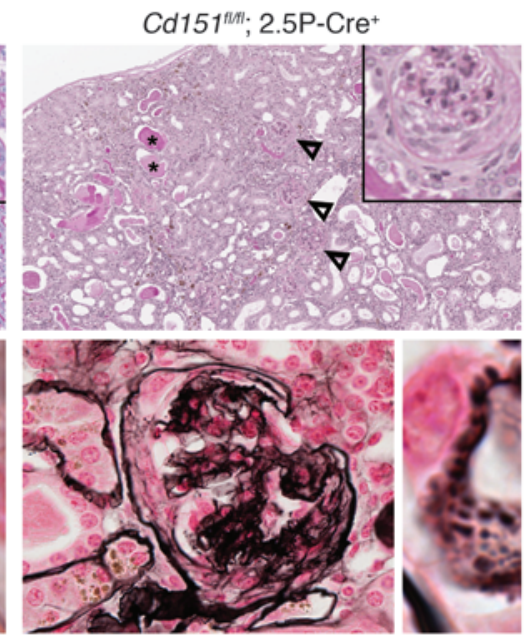
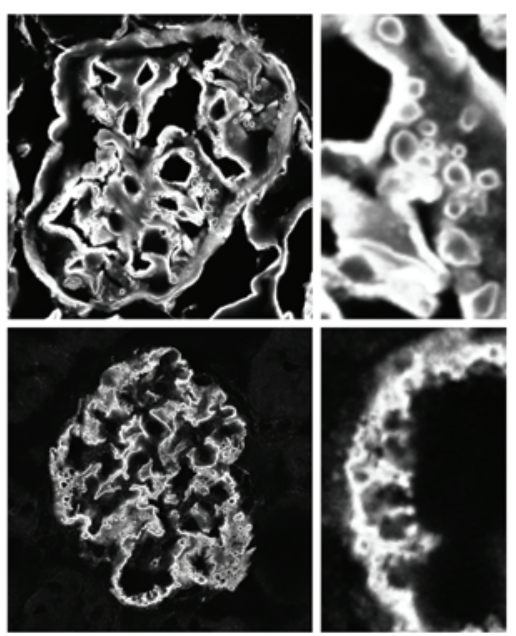

Figure 2

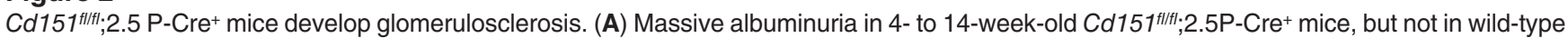
littermates. Shown is Coomassie blue-stained protein gel of $1 \mu \mathrm{l}$ urine/lane and ELISA for murine albumin present in 24-hour urine collections. (B)

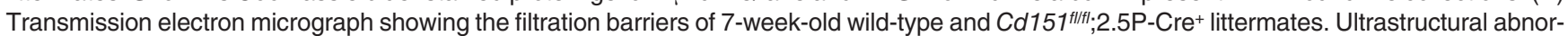
malities in the latter included abnormally broad foot processes (arrowheads) and an irregularly thickened GBM (asterisks). (C) PasD staining of the kidney cortex of a 51 -week-old $\mathrm{Cd} 151^{f|l| f \mid} ; 2.5 \mathrm{P}$-Cre+ ${ }^{+}$mouse, but not of a 71 -week-old wild-type mouse, revealed widespread glomerulosclerosis (arrowheads) and dilated tubuli containing proteinaceous casts (asterisks). Massive depositioning of GBM material was demonstrated by Jones silver staining. (D) Cryosections of glomeruli stained for nidogen and podocin to visualize GBM and podocyte abnormalities in Cd151 ${ }^{1 / 1 / 1} ; 2.5 \mathrm{P}-\mathrm{Cre}^{+}$ mice. Scale bars: $1 \mu \mathrm{m}$ (B), $500 \mu \mathrm{m}$ (C, top; insets enlarged ×2.5), $50 \mu \mathrm{m}$ (C), bottom), $20 \mu \mathrm{m}$ (D), $5 \mu \mathrm{m}$ (insets of $\mathbf{C}$, bottom, and D).

sus Cd151-negative GECs when cells were allowed to adhere onto collagen I. Therefore, CD151 did not seem to influence adhesion mediated by the collagen-binding integrin $\alpha 2 \beta 1$. To determine whether the adhesion-strengthening function of CD151 on laminin-332 is dependent on cell-cell junctions or on an intact actin cytoskeleton, we pretreated the cells with EGTA or cytochalasin D, respectively. In both cases, the absence of Cd151 resulted in approximately $35 \%$ lower shear stress tolerance (Figure 4D), similar to the reduction seen in untreated cells. Pretreating GECs with $\mathrm{Mn}^{2+}$ to activate integrins (25) could not overcome the adhesion defects of Cd151-negative GECs. Additionally, blocking the laminin-binding function of integrins $\alpha 6 \beta 1$ and $\alpha 6 \beta 4$ in Cd151-positive GECs did not significantly decrease their adhesion strength (Figure 4D). Having excluded integrin activation and $\alpha 6$-mediated podocyte adhesion as possible mechanisms for the action of
Cd151 on adhesion strengthening in our model, we hypothesized that the cell surface distribution of $\alpha 3$ might be altered. Indeed, total internal reflection fluorescence (TIRF) microscopic analysis of GECs with or without Cd151 seeded on laminin-332-rich matrix showed that $\alpha 3$ was prominently present in large focal adhesions in Cd151-negative GECs, whereas these adhesion structures were devoid of $\alpha 3$ in Cd151-positive GECs (Figure 5A). Also in vivo, $\alpha 3$ localization was visibly impaired at the podocyte-GBM interface in Cd151/flfl mice bred on a mixed B6/FVB background, which lacked albuminuria and glomerulosclerosis (Figure 5B). Thus, we concluded that Cd151 strengthens integrin $\alpha 3$-mediated adhesion of GECs in vitro, most likely by changing the distribution of $\alpha 3$ at the cell-matrix interface.

Deoxycorticosterone acetate/high-salt treatment of Cd151-null B6 mice induces albuminuria. Whether $C d 151^{-/-}$mice develop nephropathy 
A
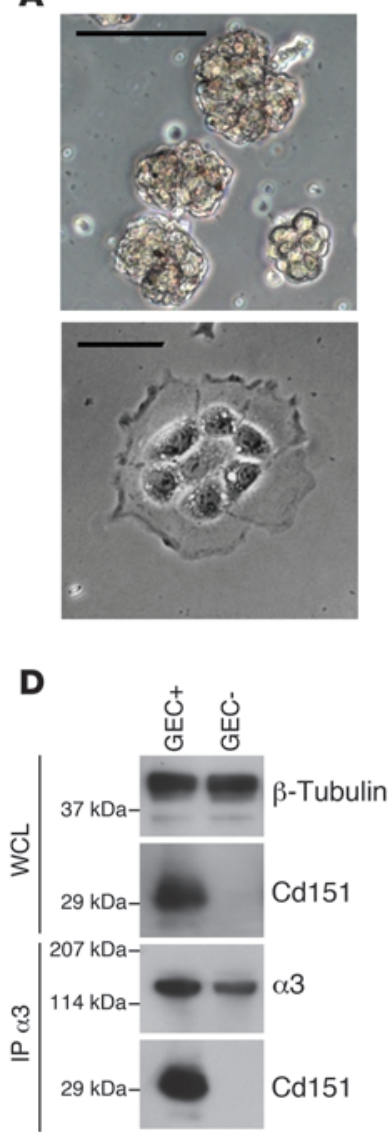

B

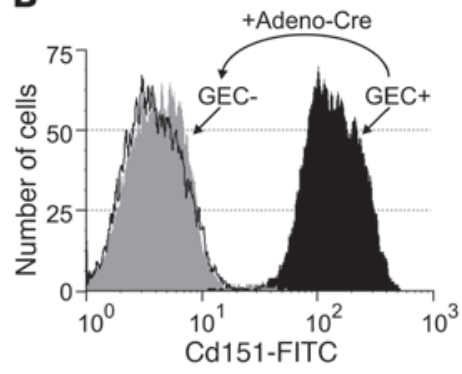

C
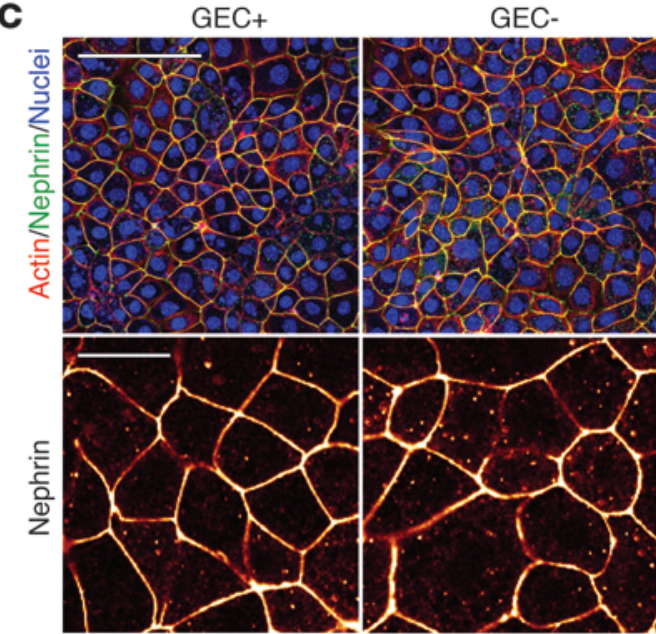

E

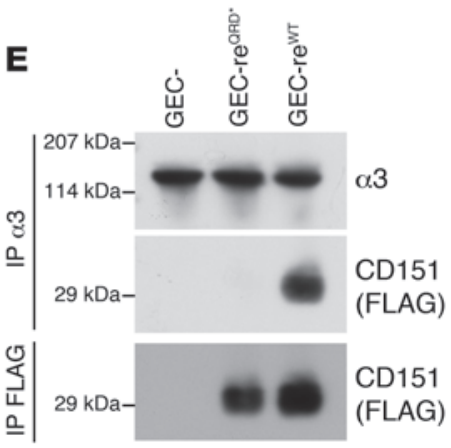

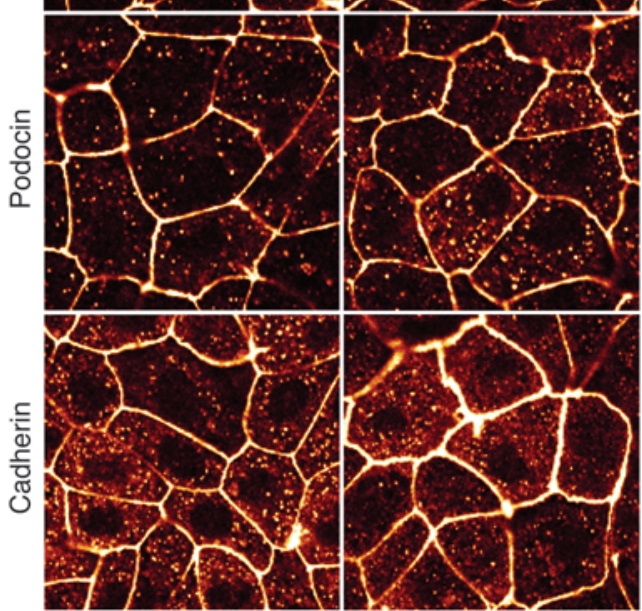

\section{Figure 3}

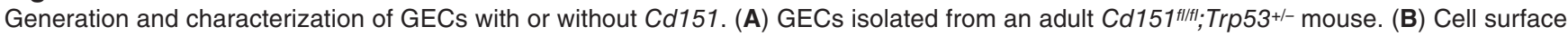
expression of Cd151 was absent after Cre-mediated recombination in vitro, as shown by FACS. GEC+, Cd151-positive GECs; GEC-, Cd151negative GECs. (C) Immunofluorescent analysis of confluent Cd151-positive and -negative GEC monolayers showing similar cell size as well as comparable actin, nephrin, podocin, and cadherin distribution. (D) Western blot analysis of whole cell lysates (WCL) showing absence of Cd151 in the Cd151-negative GECs and CD151 being coimmunoprecipitated with $\alpha 3$ in Cd151-positive, but not Cd151-negative, GECs. (E) Western blot analysis of immunoprecipitations of $\alpha 3$ and FLAG-tagged human CD151 rescue constructs. CD151 coimmunoprecipitated with $\alpha 3$ only when the QRD integrin-binding motif was intact. GEC-re ${ }^{W T}, C d 151$-negative GECs rescued with human wild-type CD151; GEC-re ${ }^{\text {QRD*, }}$ Cd151-negative GECs rescued with the mutated ${ }^{194} \mathrm{QRD}-\mathrm{INF}^{196}$ sequence of human CD151. Scale bars: $100 \mu \mathrm{m}(\mathbf{A}$, top, and $\mathbf{C}$, triple stain), $25 \mu \mathrm{m}$ (A, bottom, and $\mathbf{C}$, single stains).

greatly depends on the genetic background: absence of Cd151 causes kidney failure in mice on a mixed 129P2/FVB or FVB congenic background $(2,10)$, whereas its absence in $\mathrm{B} 6$ mice does not result in detectable kidney abnormalities $(10,11,26)$. Since lack of $C d 151$ resulted in reduced resistance to shear stress in vitro, we hypothesized that mechanical forces imposed on podocytes in vivo might differ between FVB and B6 mice. To test this further, cardiac parameters were measured under pentobarbital anesthesia in 8-week-old wild-type B6 and FVB mice. As shown in Supplemental Table 2, systolic blood pressure, heart rate, and dried heart weight moderately decreased in B6 versus FVB mice. We predicted that a systemic increase in the blood pressure of Cd151-null B6 mice would considerably increase transcapillary filtration pressure, leading to nephropathy. Thus, we next generated B6 congenic $C d 151^{-/-}$mice. In agreement with the findings from the groups of Ashman and Hemler $(10,11,26)$, these mice were healthy for 10 months and did not display any kidney abnormalities (Supplemental Figure 6). We uninephrectomized 7-week-old Cd151/t+ and Cd151-/- B6 mice and 2 weeks later implanted 60-day release pellets carrying $150 \mathrm{mg}$ deoxycorticosterone acetate (DOCA) subcutaneously in addition to offering saline for drinking water. No albuminuria was observed in either group after nephrectomy; however, after DOCA/high-salt treatment, $C d 151^{-/-} \mathrm{B} 6$ mice developed significant albuminuria, whereas $\mathrm{Cd} 151^{+/+} \mathrm{B} 6$ mice showed a modest increase in urine albumin levels (Figure 6A). Histological analysis of $\mathrm{Cd} 151^{+/+} \mathrm{B} 6$ kidneys revealed partial tubular dilation as a consequence of DOCA/high-salt treatment, but no signs of glomerulosclerosis or proteinuria. In contrast, proteinaceous casts were regularly identified in the remaining $\mathrm{Cd} 151^{-/-} \mathrm{B} 6$ kidneys after the experiment (Figure 6B). Taken together, these data showed induc- 
A

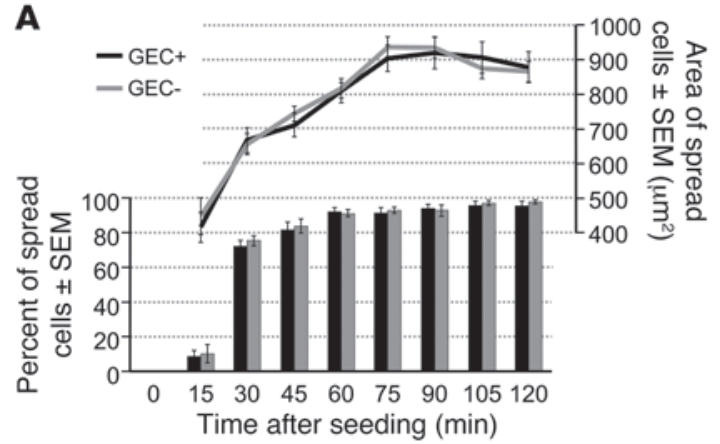

C
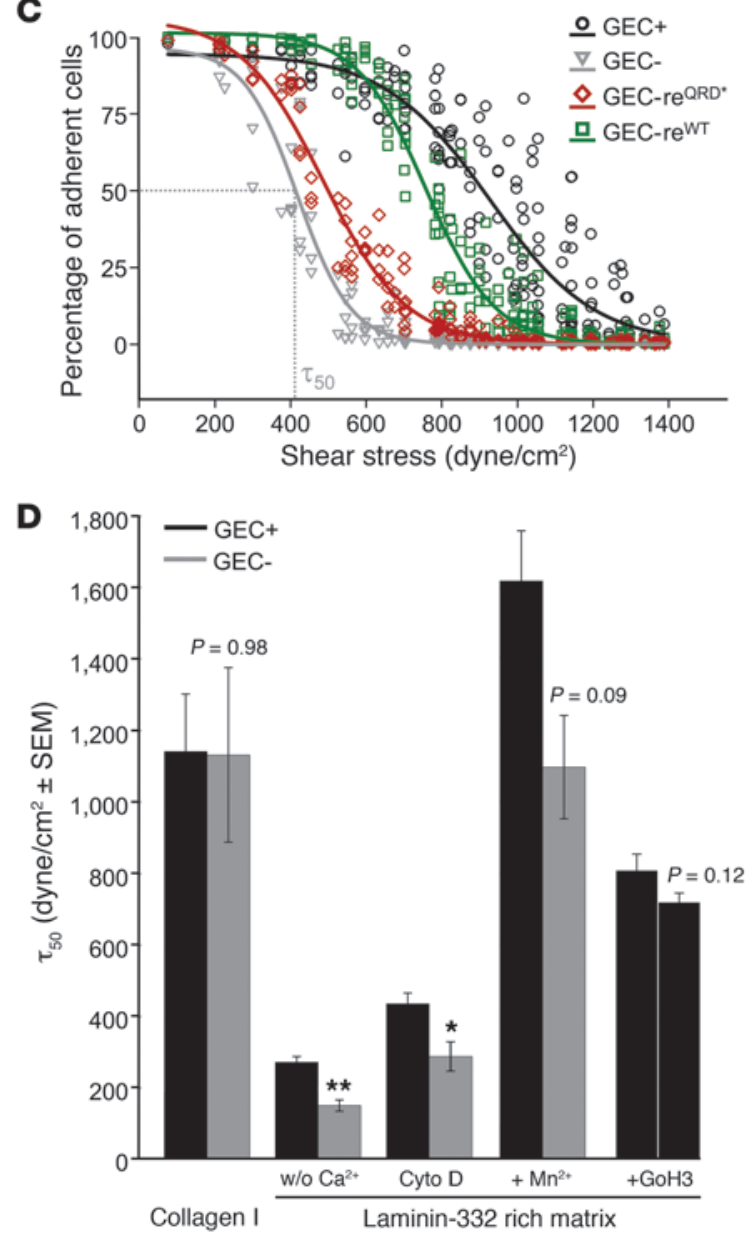
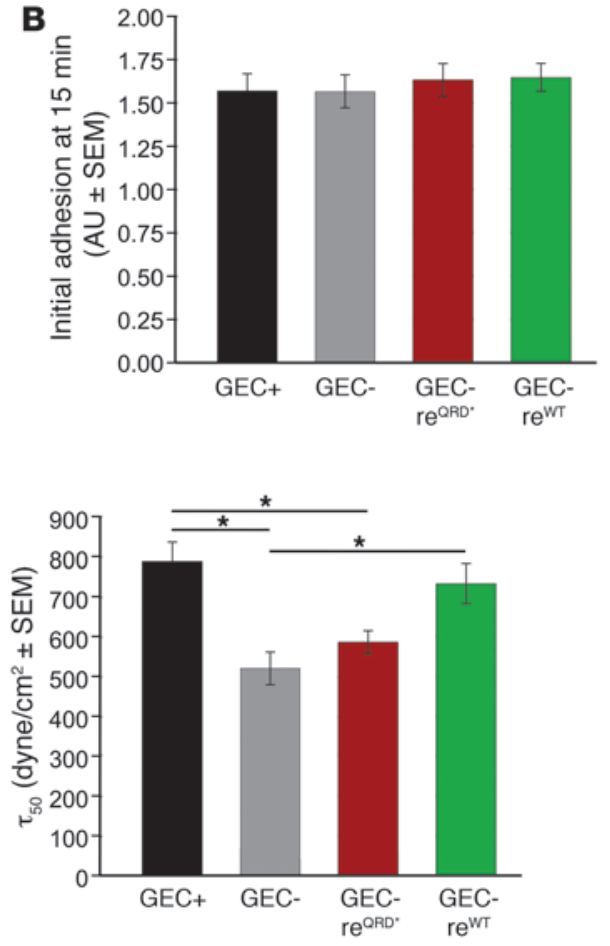

Figure 4

Spreading and adhesion assays using GECs with or without Cd151. (A) No differences were observed between Cd151-positive and -negative GECs with respect to percentage and area of spread cells on a laminin-332-rich matrix (analysis of approximately 150 cells of 2 independent experiments). (B) The presence or absence of wild-type or mutant CD151 did not affect initial adhesion of GECs to a laminin-332-rich matrix (averages of 6 independent experiments, 1-way ANOVA). (C) Adhesion strengthening assay as described by Boettiger (44). The detachment profiles for GECs with or without Cd151 adhering to laminin-332-rich matrices were fitted to sigmoid curves to obtain the shear stress values equivalent to $\tau_{50}$. Deletion of Cd151 resulted in approximately $33 \%$ lower shear stress tolerance, which was rescued upon reexpression of human wild-type CD151, but not upon expression of the integrin-binding mutant. Shown are detachment curves of a single representative experiment and averaged $\tau_{50}$ values of 5 independent experiments (1-way ANOVA). (D) The difference in adhesion strength depended on the extracellular matrix used, but not on the presence of cell-cell junctions, the actin cytoskeleton, or activated integrins. Whereas Cd151-positive and -negative GECs adhered equally strongly to $3 \mu \mathrm{g} / \mathrm{ml}$ collagen I, initially observed differences on laminin-332 were maintained after pretreatment with EGTA (w/o Ca ${ }^{2+} ; 5 \mathrm{mM}$ for 2 hours), cytochalasin $\mathrm{D}$ ( $1 \mu \mathrm{M}$ for 30 minutes), or $\mathrm{Mn}^{2+}(1 \mathrm{mM}$ in TBS for 5 minutes). Pretreatment or Cd151-positive GECs with integrin $\alpha 6$ blocking Ab GoH3 $(2 \mu \mathrm{g} / \mathrm{ml}$ for 2 hours) only marginally decreases adhesion strength (averages of 3-5 discs per condition, Student's $t$ test). ${ }^{*} P<0.05 ;{ }^{*} P<0.01$. 
A
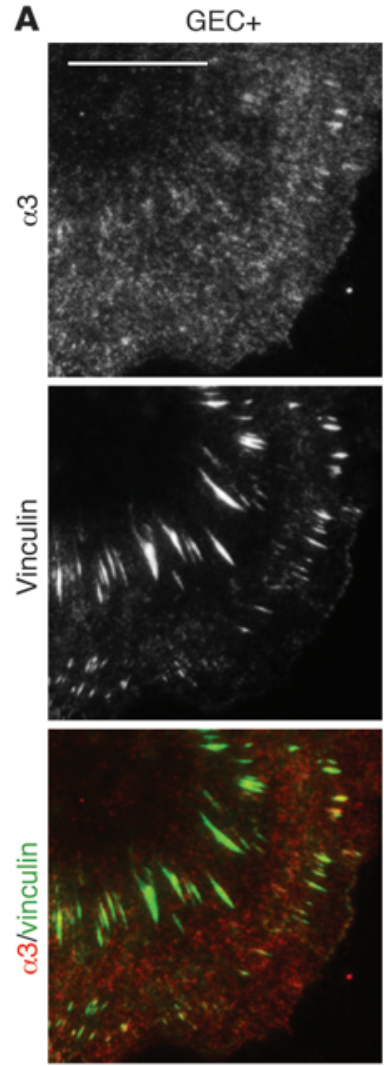

GEC-
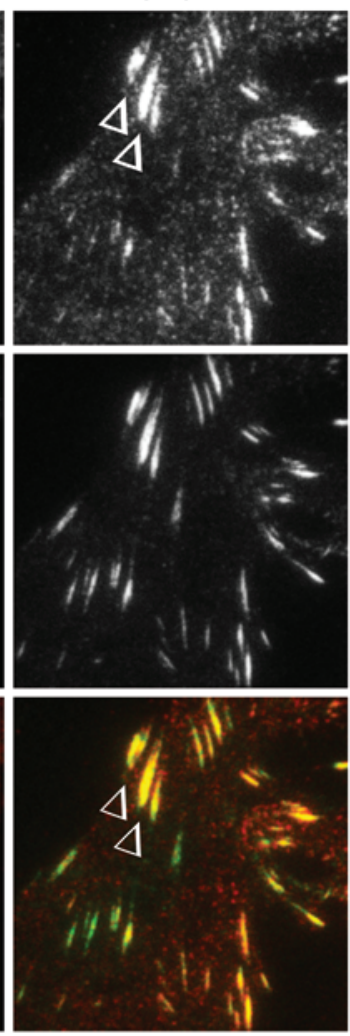

B
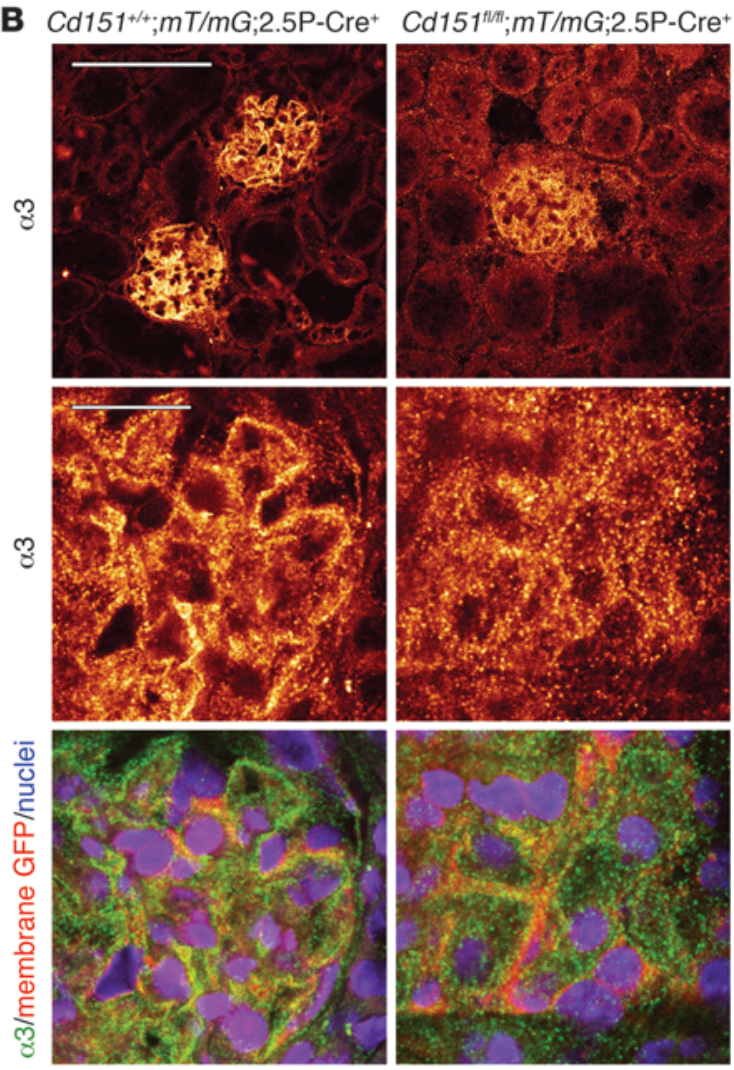

Figure 5

Cd151 influences localization of $\alpha 3$ in vitro and in vivo. (A) TIRF microscopy of GECs seeded on laminin-332 showed integrin $\alpha 3$ in large vinculinpositive focal adhesions only in the absence of Cd151 (arrowheads). (B) Absence of Cd151 in podocytes of healthy 12-week-old B6/FVB mice led to diffuse membranous distribution of $\alpha 3$. Recombination of the $m T / m G$ reporter allele and subsequent expression of membrane-targeted GFP was indicative of Cre activity and outlined podocyte membranes. Scale bar: $100 \mu \mathrm{m}$ (B, top row), $20 \mu \mathrm{m}$ (A and B, bottom rows).

tion of albuminuria in normally resistant $C d 151^{-1-} \mathrm{B} 6$ mice by systemically increasing blood pressure.

Blocking the angiotensin-converting enzyme in Cd151-null FVB mice prolongs survival. We next hypothesized that reducing blood and intraglomerular pressure would diminish the mechanical stress experienced by podocytes and possibly mitigate $C d 151$ knockoutinduced nephropathy. Blood pressure-lowering drugs, such as calcium-channel blockers (e.g., verapamil) and angiotensin-converting enzyme (ACE) inhibitors (e.g., enalapril), have previously been shown to successfully reduce renal injury in experimental models. Although both classes of drugs systemically reduce blood pressure, only ACE inhibitors reduce intraglomerular pressure $(27,28)$. Here, we provided $\mathrm{Cd} 151^{-/} \mathrm{FVB}$ mice verapamil or enalapril in mother's milk and drinking water and compared their kidney function with that of untreated $\mathrm{Cd} 151^{-/-} \mathrm{FVB}$ mice. Mice were sacrificed and analyzed after they had lost more than $15 \%$ of their body weight and/or showed signs of intraperitoneal edema. The Kaplan-Meier curve in Figure 6C shows that median survival did not significantly differ between mice of the control and verapamil-treated groups (27 and 21 weeks, respectively). In contrast, median survival of enalapriltreated $\mathrm{Cd} 151^{-/}$FVB mice was significantly prolonged (46 weeks; $P<0.01, \log$-rank test). Histological analysis of kidneys from nearly age-matched $C d 151^{-/-} \mathrm{FVB}$ mice treated or not with enalapril showed proteinaceous casts, tubular dilation, and severe glomeru- losclerosis without treatment and almost no abnormalities in the enalapril-treated group $(n=2$; Figure $6 \mathrm{D})$. To test whether both drugs affected blood pressure to the same extent, we measured blood pressure in awake 5- to 7-week-old mice from the 3 groups. Indeed, both enalapril and verapamil lowered the systemic blood pressure of $C d 151^{-/-}$FVB mice approximately $10 \%$ compared with untreated mice (Supplemental Table 3). These results showed that lowering the blood pressure of susceptible $C d 151^{-/-}$FVB mice can systematically delay nephropathy and increase median survival by $70 \%$ when a drug is used that also affects intraglomerular pressure (e.g., the ACE inhibitor enalapril).

\section{Discussion}

Previous studies have shown that humans with mutated CD151 and mice lacking $C d 151$ in all tissues develop renal failure $(2,10$, 12). However, several questions regarding cell type and the mechanism responsible for the underlying pathology, as well as possible therapeutic intervention, have remained unanswered. The results of the present study showed that in human podocytes, CD151 bound integrin $\alpha 3 \beta 1$ in vivo after the early capillary loop stage. CD151 was not obviously enriched in cell-cell junctions, as has been observed previously in several cell lines (29-31), but instead was localized ultrastructurally at the podocyte-GBM interface. Even though renal expression of CD151 was strongest in podo- 
A
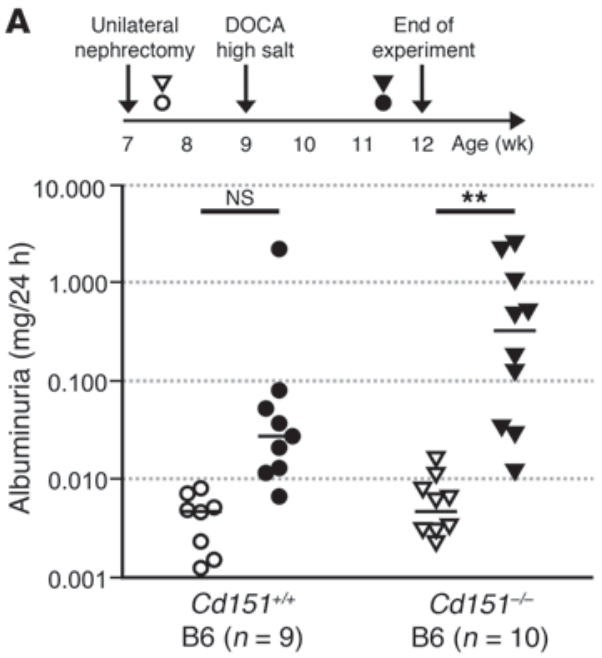

C

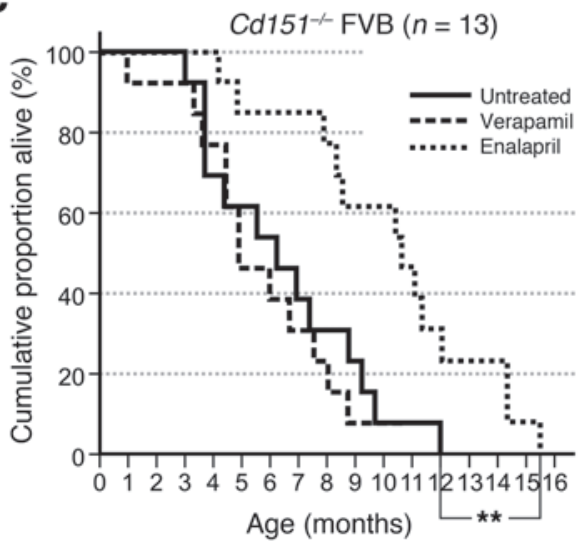

B Nephrectomized kidney

Kidney after experiment
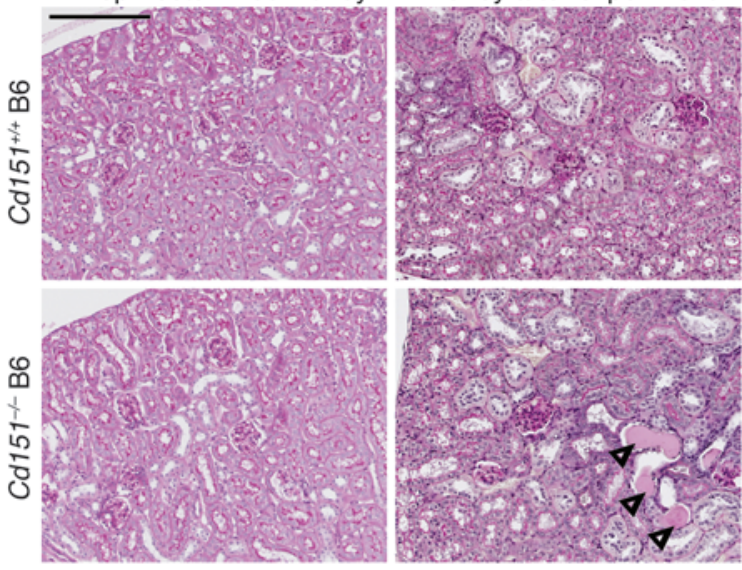

D
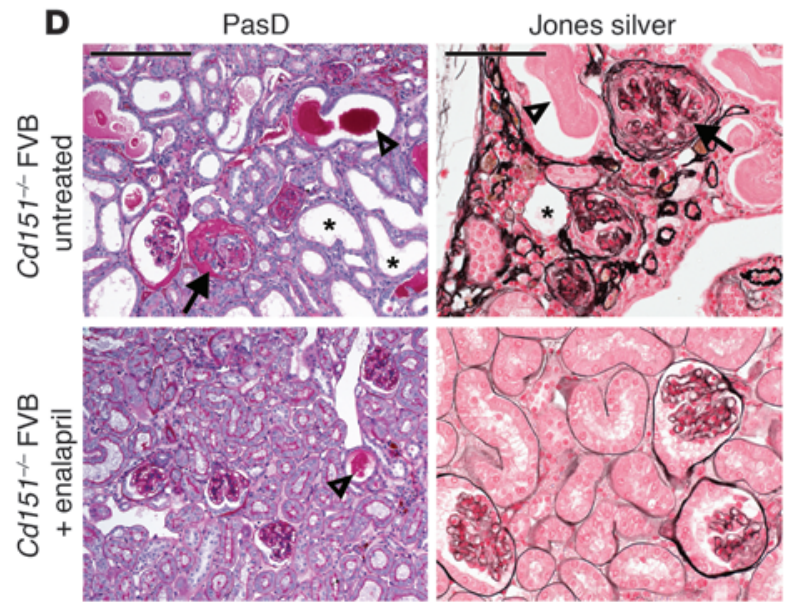

\section{Figure 6}

Altering blood and intraglomerular pressure of mouse strains susceptible (FVB) or not (B6) to Cd151 knockout-induced nephropathy greatly influences renal pathology. (A) Uninephrectomized Cd151-- B6 mice with DOCA/high-salt-induced hypertension developed albuminuria, whereas (apart from 1 outlier) $\mathrm{Cd151+/+} B 6$ mice did not, as quantified by ELISA ( $P<0.001$ vs. $P=0.051$, Mann-Whitney $U$ test). Symbols are as in the experimental schematic above. (B) PasD-stained wild-type and Cd151-- B6 kidney cortices before and after DOCA pellet implantation and high-salt diet. Proteinaceous casts (arrowheads) were present exclusively in tubuli of the remaining Cd151 ${ }^{-/}$B6 kidney. (C) Decreasing blood pressure of susceptible Cd151-/- FVB mice by lifelong oral administration of verapamil (20 mg/l) did not significantly alter median survival (27 weeks vs. 21 weeks in untreated mice; $P=0.52$, log-rank test). In contrast, decreasing blood and intraglomerular pressure by lifelong oral administration of enalapril $(20 \mathrm{mg} / \mathrm{l})$ prolonged median survival significantly (46 weeks; $P=0.0068$ vs. untreated, log-rank test). (D) Renal histopathology of a 19-week-old untreated and an 18-week-old enalapril-treated mouse. At this age, untreated mice showed signs of massive proteinuria (arrowheads), tubular dilation (asterisks), and glomerulosclerosis (arrow), whereas treated mice displayed occasional proteinaceous casts (arrowhead). Scale bars: $200 \mu \mathrm{m}$ (B and D, left), $100 \mu \mathrm{m}$ (D, right). ${ }^{* *} P<0.01$.

cytes, one cannot formally attribute the renal failure of Cd151-null mice to these cells exclusively, because CD151 is also present in the glomerular endothelium as well as the tubular epithelium (19). We therefore generated podocyte-specific Cd151-knockout mice $\left(C d 151^{f l f l} ; 2.5 \mathrm{P}-\mathrm{Cr} \mathrm{e}^{+}\right)$, which showed the same phenotype as $C d 151^{-/-}$ mice. We conclude that the presence of Cd151 in podocytes is critically important for renal homeostasis after the S-shape body stage, during which podocytes start to differentiate and 2.5P-Cre expression is driven (20). In contrast, there were different kidney phenotypes in the corresponding mouse models lacking $\alpha 3$ : total Itga3-null animals showed developmental defects in glomeruli, proximal tubules, and collecting ducts, whereas podocyte-specific Itga3-knockout mice displayed abnormalities in the GBM and podocytes only $(2,15)$. The latter phenotype is largely identical to that of $C d 151^{f l / f l} ; 2.5 \mathrm{P}-\mathrm{Cre}^{+}$mice and shows that $\mathrm{Cd} 151-\alpha 3 \beta 1$ complexes are functionally relevant during and after podocyte differentiation. In situ PLA on fetal human kidneys revealed that the expression of CD151- $\alpha 3 \beta 1$ complexes dramatically increased during the capillary loop stage. It is therefore tempting to speculate that strong adhesion mediated by CD151- $\alpha 3 \beta 1$ complexes is required only during later stages of glomerulogenesis, when differentiated podocytes have to withstand transcapillary filtration pressure. Deletion of Itgb4 in podocytes did not result in glomerular abnormalities, as has been suggested previously for a focal segmental glomerulosclerosis patient carrying a homozygous missense mutation in ITGB4 (22). Although we cannot rule out 
that $\beta 4$ expression prior to Cre-mediated excision during S-shape body stage has a certain function, these mouse models suggest that the interaction of $\mathrm{Cd} 151$ with $\alpha 3 \beta 1$, rather than with $\alpha 6 \beta 4$, is essential for podocyte function. However, $\alpha 6$ can also associate with $\beta 1$, and, even though the expression of $\alpha 6 \beta 1$ has previously been shown to be restricted to tubuli (32), the results of podocytespecific deletion of Itga 6 will be necessary to formally exclude $\alpha 6$ as a podocyte adhesion receptor in vivo.

To investigate whether and how Cd151 influences $\alpha 3 \beta 1$-mediated adhesion, we generated GECs with or without $C d 151$. Whereas initial adhesion to the $\alpha 3 \beta 1$ ligand laminin-332 was independent of Cd151, the strength of adhesion was significantly weaker in Cd151negative GECs. The fact that adhesion strengthening by Cd151 occurred on laminin-332, but not on collagen I ( $\alpha 2 \beta 1$ ligand), is in line with the prior observation that CD151 mainly interacts with laminin-binding integrins (32). Importantly, we were able to rescue the defect in adhesion strengthening of Cd151-deficient GECs with wild-type CD151, but not with a CD151 mutant incapable of binding $\alpha 3$. The contribution of $\alpha 6$ to podocyte adhesion seems minor, as pretreatment of GECs with the function-blocking Ab GoH3 barely reduced adhesion strengthening. Although it has previously been suggested that CD151 alters the affinity of $\alpha 3 \beta 1$ $(18,32)$, this does not seem to be the case in our system, because spreading and initial adhesion on laminin-332 was not different in GECs with or without Cd151. We were furthermore unable to rescue the weaker adhesion strength of Cd151-negative GECs to laminin by activating integrins with $1 \mathrm{mM} \mathrm{Mn}^{2+}$. In addition, we ruled out cell-cell junctions and the actin cytoskeleton as effectors of the adhesion-strengthening function of CD151. However, we observed varied distributions of $\alpha 3$ depending on the presence of CD151. Because in the Cd151-positive GECs, $\alpha 3 \beta 1$ was not clustered in focal adhesions, but diffusely distributed over the entire basal cell surface, adhesions of these cells to the extracellular matrix occurred over relatively large surface areas. This was in contrast to Cd151-negative GECs, in which $\alpha 3 \beta 1$ interacted with the substratum solely through focal adhesions. Clustering of $\alpha 3 \beta 1$ into focal adhesions in Cd151-positive GECs did not occur, because its association with Cd151 most likely led to its incorporation into a tetraspanin web (33). We suggest that the much larger surface area over which Cd151-positive GECs interacted with the substratum is fundamental for the increased adhesion strength of these cells compared with Cd151-negative GECs. In line with this assumption, we also observed altered subcellular localization of $\alpha 3$ in vivo. While in the presence of CD151, the distribution of $\alpha 3$ was essentially continuous with GBM, in its absence, the distribution appeared to be more punctuated.

Importantly, loss of Cd151 did not necessarily lead to renal failure: kidneys of Cd151-null mice on a B6 background were functionally and histologically normal for up to 12 months of age, in agreement with the literature (10). In contrast, $C d 151^{-/-} \mathrm{FVB}$ mice developed the severe glomerular disease previously described by us and others $(2$, 10). In order to study the development and progression of nephropathy in these mice, we altered the mechanical stress imposed on podocytes experimentally. DOCA/high-salt-induced hypertension in Cd151-null B6 mice caused proteinuria and proteinaceous casts, whereas wild-type mice remained healthy. Decreasing blood as well as glomerular filtration pressure in Cd151-null FVB mice with the ACE inhibitor enalapril significantly prolonged their median life span compared with that of untreated controls. Conversely, antihypertensive treatment with the calcium entry blocker verapamil decreased blood pressure systemically, but did not affect glomerular hypertension, probably as a result of increased plasma renin levels and efferent arteriolar vasoconstriction (27). In line with our model of Cd151-mediated adhesion strengthening in podocytes, we did not observe a beneficial effect on survival of $\mathrm{Cd} 151^{-/-} \mathrm{FVB}$ mice treated with verapamil. We therefore established that $C d 151$ deficiency-induced nephropathy cannot be modified by altering blood pressure alone, but must target intraglomerular pressure.

In conclusion, we present evidence that podocyte CD151 binds integrin $\alpha 3$ in situ and increases its adhesion strength to laminin through predicted changes in subcellular localization. We further suggest that Cd151-null nephropathy is caused by the lack of $\mathrm{Cd} 151$ in podocytes and can be treated by blocking ACE.

\section{Methods}

Abs. Mouse mAbs used were 11B1.G4 against CD151 (34), TS2/16 against integrin $\beta 1$ (35), 29A3 against $\alpha 3 \mathrm{~A}$ (36), and hVIN-1 against vinculin (V9131; Sigma-Aldrich). Rat mAbs used were MAB1556 against mouse podocalyxin (clone 192703), MAB4609 against mouse Cd151 (R\&D Systems Inc.), GoH3 against $\alpha 6$ (37), MB1.2 against $\beta 1$ (38), 346-11A against $\beta 4$ (BD), and 390 against Cd31 (gift from C. Buck, Wistar Institute, Philadelphia, Pennsylvania, USA; ref. 39). Rabbit polyclonal Abs used were 141742 against $\alpha 3 \mathrm{~A}$ (2), 140190 against Cd151 (2), anti-mouse nidogen (gift from T. Sasaki, Max Planck Institute for Biochemistry, München, Germany), anti-mouse podocin (gift from C. Antignac, Cochin Biomedical Research Institute, Paris, France), anti-nephrin and anti-podocin (both gifts from H. Holthöfer, University of Helsinki, Helsinki, Finland), anti-pan-cadherin (C3678; Sigma-Aldrich), and D-8 against FLAG (sc-807; Santa Cruz Biotechnology Inc.). Goat Abs used were N20 against WT1 (sc-15421; Santa Cruz Biotechnology Inc.) and AF2787 against mouse $\alpha 3$ (R\&D Systems Inc.). Hamster anti-mouse $\alpha 2$ (Hma2) was from BD. Secondary Abs coupled to FITC, Texas Red, Cy5, and horseradish peroxidase were obtained from Jackson ImmunoResearch Laboratories.

Immunofluorescent and in situ PLA analysis. Adult and fetal (17-23 weeks gestational age) human kidneys were studied. $4-\mu \mathrm{m}$-thick cryostat sections were cut, dried, and acetone fixed. For immunofluorescence analysis, we used primary Abs 11B1.G4 (anti-CD151, diluted 1:500) and 141742 (anti$\alpha 3 \mathrm{~A}$, diluted 1:200) diluted in normal Ab diluent (Immunologic; Klinipath) in overnight incubations. IgG-specific secondary Abs coupled to FITC or Texas Red were diluted 1:200 and incubated for 1 hour. Sections were mounted in Vectashield containing DAPI (Vector Laboratories). In situ PLA (Duolink PLA; Olink Bioscience) was performed according to the manufacturer's instructions. Primary Abs were 11B1.G4, 141742, TS2/16, or the respective IgG control; secondary Abs conjugated with oligonucleotides were DuoLink anti-rabbit PLUS and DuoLink anti-mouse MINUS; and the detection kit was either DuoLink 613 or Duolink II Brightfield, with DuoLink Mounting Medium containing Hoechst or Pertex, respectively. Epifluorescence imaging was performed using a Leica DM5000B microscope with PL FLUOTAR 40×/1.00-0.50 and HCX PL APO 63×/1.40-0.60 oil immersion objectives. Images were recorded using a Leica DFC 500 camera and Leica Application Suite Imaging software (Leica Microsystems). In addition, analysis of multiple stained slides was performed with spectral imaging using a Nuance N-MSI-420-20 camera with Nuance 3.0.0 software (Cambridge Research and Instrumentation). Data sets were acquired from 440 to $500 \mathrm{~nm}$ for A4, from 500 to $720 \mathrm{~nm}$ for K3, and from 580 to $720 \mathrm{~nm}$ for N2.1 filter blocks, each at 10-nm intervals. Spectral libraries either for single-DAPI/-FITC/-Texas Red or for single-Hoechst 33342/-DuoLink 613 were used to split the triple or double staining signals into the individual channels and to separate them from autofluorescence. Nuance software was used to construct composite images. 
Mouse kidneys were excised and embedded in cryoprotectant (TissueTek O.C.T.; Sakura Finetek Europe). Cryosections were prepared, fixed in ice-cold acetone, and blocked with $2 \%$ BSA in PBS. Cells were fixed for 10 minutes in $2 \%$ PFA, permeabilized with $0.05 \%$ Triton X-100, and blocked with $2 \%$ BSA in PBS. Primary Abs were incubated for 60 minutes at 1:100 (anti-podocin, anti-nephrin), 1:200 (anti-vinculin, anti-pan-cadherin), and 1:500 (anti-nidogen) dilutions in 2\% BSA in PBS, followed by incubation with secondary Abs diluted 1:200 for 60 minutes. Samples were analyzed at $37^{\circ} \mathrm{C}$ using either a $63 \times / 1.4 \mathrm{HCX}$ PL APO CS oil objective on a TCS SP2 AOBS confocal microscope (Leica Microsystems GmbH) or a $63 \times / 1.4 \mathrm{PL}$ APO DIC ACR oil objective on a Zeiss Axio Observer Z1 TIRF microscope (Carl Zeiss MicroImaging Inc.). Images were acquired using LCS 2.61 (Leica Microsystems $\mathrm{GmbH}$ ) or a cooled Hamamatsu ORCA R2 black and white CCD camera with AxioVision 4.8.1 software (Carl Zeiss MicroImaging Inc.) and processed using Adobe Photoshop CS4 or ImageJ.

Histological analyses. Mouse kidneys were excised, fixed for 1 day in formaldehyde, embedded in paraffin, sectioned, and stained with PAS-D, H\&E, or Jones methenamine silver. Images were taken with PL APO objectives (10×/0.25 NA, $40 \times / 0.95$ NA, and 63×/1.4 NA oil) on an Axiovert S100/ AxioCam HR color system using AxioVision 4 software (Carl Zeiss MicroImaging Inc.) or with a $20 \times / 0.75$ NA PL APO objective with or without a $2 \times$ optical mag changer on a ScanScope XT system using ImageScope version 10 software (Aperio Technologies Inc.).

Ultrastructural analyses. After fixation in Karnovsky buffer for 48 hours, mouse kidneys were postfixed with $1 \%$ osmiumtetroxide, block-stained with $1 \%$ uranyl acetate, dehydrated in ethanol series, and embedded in epoxyresin Embed-812 (Agar Scientific Ltd). Tissue sections were stained with uranyl acetate and lead oxide, then examined using a transmission electron microscope (Philips CM10; FEI). Human kidney for immunoelectron microscopy was fixed overnight in 1\% PFA in $0.1 \mathrm{M}$ PHEM buffer $(60$ mM PIPES, 25 mM HEPES, 2 mM MgCl 2,10 mM EGTA, pH 6.9). 50-nm cryosections were cut at $-115^{\circ} \mathrm{C}$ using diamond knives in a cryoultramicrotome (Leica AG) and transferred with a mixture of sucrose and methylcellulose onto formvar-coated copper grids. The grids were placed on $35-\mathrm{mm}$ Petri dishes containing $2 \%$ gelatin. Ultrathin frozen sections were incubated at room temperature with 11B1.G4 (anti-CD151, diluted 1:100) followed by a rabbit anti-mouse bridging $\mathrm{Ab}$ and then incubated with $10-\mathrm{nm}$ protein $\mathrm{A}-$ conjugated colloidal gold (EM Lab, Utrecht University, Utrecht, The Netherlands). After immunolabeling, the sections were embedded in a mixture of methylcellulose and uranyl acetate and examined with a Philips CM10 electron microscope (FEI Co.).

Animal experiments. All mice used in this study were housed at 1-5 animals per cage in a room with a 12-hour/12-hour artificial light cycle (7:00 am to 7:00 pm), a temperature of $21^{\circ} \mathrm{C} \pm 1^{\circ} \mathrm{C}$, and humidity of $55 \% \pm 5 \%$. The animals had free access to standard chow and drinking water throughout the experiment.

$C d 151^{f / f l}$ mice were generated in parallel to $C d 151^{-/-}$mice described previously (2), without Cre-mediated recombination of loxP sites in targeted embryonic stem cells. Mice were backcrossed onto an FVB background for more than 10 generations and crossed with 2.5P-Cre FVB (N6) mice (20) to generate $C d 151^{f / f l} ; 2.5 \mathrm{P}-\mathrm{Cre}{ }^{+}$mice. Conditional Itgb4 knockout and $m T / m G$ reporter mice have been previously described $(40,41)$ and were backcrossed onto FVB for 8 and 4 generations, respectively, before intercrossing them with 2.5P-Cre mice. Cd151/- mice on a mixed 129P2/FVB background (2) were backcrossed 16 and 9 times to FVB and B6 to generate FVB.129P2Cd151<tm1Son>/A and B6.129P2-Cd151<tm1Son>/A, respectively.

To induce hypertension in B 6 mice, the anesthetized animals underwent right unilateral nephrectomy at 7 weeks of age followed by subcutaneous implantation of a 60-day release pellet containing $150 \mathrm{mg}$ DOCA (IRA), and drinking water was switched from tap to saline 2 weeks later. To decrease blood pressure in $\mathrm{Cd} 151^{-/-} \mathrm{FVB}$ mice, pregnant $\mathrm{Cd} 151^{+/-}$mothers were treated with $20 \mathrm{mg} / \mathrm{l}$ enalapril maleate or $80 \mathrm{mg} / \mathrm{l}$ verapamil hydrochloride from E17 on (Sigma-Aldrich). Pups older than 3 weeks were offered drinking water supplemented with $20 \mathrm{mg} / \mathrm{l}$ enalapril maleate or $20 \mathrm{mg} / \mathrm{l}$ verapamil hydrochloride until termination of the experiment. For urine analysis, mice were placed in metabolic cages for 24 hours, and urine samples were analyzed by SDS-PAGE, followed by Coomassie Brilliant blue staining or by competitive albumin ELISA (Exocell). Blood pressure measurements of wild-type B6 and FVB mice were carried out on pentobarbital-anesthetized $(60 \mathrm{mg} / \mathrm{kg})$ mice using a murine tail-cuff system (ADInstruments). Systolic blood pressure and heart rate were averaged from 6 consecutive measurements during a 10-minute period. Blood pressure measurements of awake Cd151 $1^{-/-} \mathrm{FVB}$ mice treated or not with enalapril or verapamil were carried out on a CODA 2 system for noninvasive blood pressure measurements for mice and rats (emka technologies). 5- to 7-week-old mice were measured by averaging at least 5 consecutive measurements in a 20-minute period.

Glomeruli were isolated from an adult anesthetized $C d 151^{f l / f l}$; $\operatorname{Tr} p 53^{+/-}$ mouse as previously described using dynabead perfusion (23).

Generation of GECs. GECs grew out of isolated glomeruli in keratinocyte serum-free medium (17005; Gibco, Invitrogen) and were sorted 3 times by fluorescence-activated cell sorting (FACS) for strong expression of podocalyxin, followed by 1 negative sort for $\mathrm{Cd} 31$. Adeno-Cre (obtained from F. Graham, McMaster University, Hamilton, Ontario, Canada; ref. 42) was used to delete $C d 151$ and generate the $C d 151$-negative GEC line. Absence of Cd151 on the cell surface was confirmed by FACS. Retroviral expression constructs carrying wild-type and ${ }^{194} \mathrm{QRD}-\mathrm{INF}{ }^{196} \mathrm{CD} 151$, used to rescue expression of CD151 in Cd151-negative GECs, were from M. Hemler (DanaFarber Cancer Institute, Boston, Massachusetts, USA; ref. 24). Cells were FACS sorted for equal cell surface expression of CD151 using 11B1.G4.

FACS. Cells were trypsinized, washed with $2 \%$ FCS in PBS, and stained with primary Abs against podocalyxin and $\beta 4$ (diluted 1:200); Cd151, $\alpha 2$, and $\alpha 3$ (diluted 1:100); and Cd31, $\alpha 6$, and $\beta 1$ (undiluted) for 60 minutes on ice. After washing, secondary anti-rat, -goat, and -hamster Abs coupled to FITC were used at 1:200 dilution for 60 minutes on ice. Cells were strained and analyzed on a 1998 BD FACSCalibur (BD) using a $488 \mathrm{~nm}$ laser and a 530/30 FL1 filter configuration.

Immunoblotting and immunoprecipitations. For biochemical assays, cells were lysed in $1 \%$ (vol/vol) Nonidet P-40; 20 mM Tris-HCl, pH 7.6; 4 mM EDTA; and $100 \mathrm{mM} \mathrm{NaCl}$ supplemented with a cocktail of protease inhibitors (P8340; Sigma-Aldrich). Lysates were cleared by centrifugation for 20 minutes at $20,000 \mathrm{~g}$ at $4{ }^{\circ} \mathrm{C}$, followed by separation of proteins on $4 \%-12 \%$ polyacrylamide gels under nonreducing conditions (NuPage; Invitrogen) and transfer to Immobilon PVDF membranes (EMD Millipore). For immunoprecipitations, lysates were incubated overnight with mAb 29A3 coupled to $\gamma$-bind sepharose (GE Healthcare) or mAb M2-coupled agarose (A2220; Sigma-Aldrich). Beads were spun down at 500 $\mathrm{g}$, washed with lysis buffer and PBS, and processed by SDS-PAGE as described above. After Western blotting, membranes were blocked, and blots were developed with the indicated Abs using an ECL detection kit (GE Healthcare) according to the manufacturer's protocol.

Spreading and adhesion assays. Matrices used for adhesion assays were collagen I ( 30 minutes coating at $37^{\circ} \mathrm{C}$ using $3 \mu \mathrm{g} / \mathrm{ml}$ rat tail collagen I in PBS; Inamed Biomaterials) and a laminin-332-rich matrix deposited by Rac11-P cells (43). Briefly, cells were grown to confluency overnight; detached in $20 \mathrm{mM}$ EDTA, $0.05 \%$ sodium azide, and $0.1 \%$ protease inhibitor cocktail (P8340; Sigma-Aldrich) in PBS; and blocked with 0.5\% BSA in PBS.

For cell spreading assays, Cd151-positive and -negative GEC lines were incubated with green and orange CellTracker (C2925 and C2927; Invitrogen), washed extensively, harvested by trypsinization, allowed to recover for 30 minutes at $37^{\circ} \mathrm{C}$ in IMDM (Sigma-Aldrich) containing $0.35 \%$ BSA, mixed in a 1:1 ratio, and seeded onto coated $24-w e l l$ plates at 20,000 cells/well. Spread- 
ing was observed at $37^{\circ} \mathrm{C}$ on a temperature-controlled Zeiss Axiovert $200 \mathrm{M}$ inverted microscope using a $10 \times / 0.25$ Achroplan $\mathrm{Ph} 1$ objective in combination with a 1.6x Optovar. Images were acquired every 15 minutes using an AxioCam MRm Rev.2 black and white camera and AxioVision Rel. 4.7.2 (Carl Zeiss MicroImaging Inc.) and quantified using ImageJ.

For short-term adhesion assays, cells were trypsinized, allowed to recover for 30 minutes at $37^{\circ} \mathrm{C}$ in IMDM supplemented with $0.35 \%$ BSA, and seeded onto coated 96 -well plates at $10^{5}$ cells/well in triplicate. After 15 minutes of incubation at $37^{\circ} \mathrm{C}$, medium and nonadherent cells were discarded by vigorous tapping of the inverted plate. Adherent cells were fixed in $2 \%$ PFA and stained with $5 \mathrm{mg} / \mathrm{ml}$ crystal violet in 2\% ethanol (C3886; SigmaAldrich). Absorbance was quantified at $655 \mathrm{~nm}$ using an iMark microplate reader (Bio-Rad Laboratories).

Adhesion strengthening assays were carried out on a home-built spinning disc machine as described by Boettiger (44). $10^{6}$ cells were seeded onto coated 30 -mm glass coverslips; 20 hours later, these were subjected to a varying range of shear stresses in (Dulbecco) PBS or TBS for 5-10 minutes at room temperature. When necessary, 2.5\% 500-MDa dextran (Sigma-Aldrich) was added to increase viscosity. When required, cells were pretreated for 2 hours with $5 \mathrm{mM}$ EGTA, for 30 minutes with $1 \mu \mathrm{M}$ cytochalasin D (SigmaAldrich), or for 5 minutes with $1 \mathrm{mM} \mathrm{MnCl}_{2}$. Adherent cells were fixed in $2 \%$ PFA, stained with DAPI dihydrochloride (Sigma-Aldrich), and imaged on an automated AxioObserver Z1 inverted microscope using a 5×/0.15 Plan-Neofluar objective, a Hamamatsu ORCA-ER black and white CCD camera, and AxioVision Rel. 4.7.2 (Carl Zeiss). ImageJ was used to quantify the proportion of detached cells per microscopic field. Sigmoidal detachment curves for determination of $\tau_{50}$ were generated using SigmaPlot 11 (Systat).

Statistics. When comparing 2 groups, statistical significance was determined using 2-tailed Student's $t$ test or Mann-Whitney $U$ test as indicated (SPSS 16). The statistical significance of experiments involving more than 2 groups was determined by 1 -way ANOVA followed by Bonferroni posthoc multiple-comparison tests (SPSS 16). Survival analysis was carried out using the log-rank test (Prism 5; GraphPad). $P$ values less than 0.05 were considered statistically significant.

Study approval. Subjects or their relatives approved the use of remaining body material for education or research purposes after completion of its use for diagnostic purposes. Because material was anonymized and coded, specific informed consent was not required. Studies using human tissue were conducted according to the Code for Proper Secondary Use of Human Tissues of the Dutch Federation of Medical Research Associations (www. federa.org) and the AMC Research Code and were not subject to approval of the Medische Ethische Toetsings Commissie of AMC. All mouse experiments were approved by the Animal Experiments Review Board of The Netherlands Cancer Institute, in compliance with Dutch legislation for the care and use of laboratory animals.

\section{Acknowledgments}

The authors are grateful to C. Antignac, C. Buck, B. Chan, F. Graham, M. Hemler, H. Holthöfer, L. Holzmann, and T. Sasaki for providing Abs, plasmids, and 2.5P-Cre mice. We thank all employees of the NKI-AvL mouse facility, mouse histology department, technical department, and digital microscopy for their excellent technical assistance.

Received for publication May 5, 2011, and accepted in revised form October 12, 2011.

Address correspondence to: Arnoud Sonnenberg, The Netherlands Cancer Institute, Division of Cell Biology, Plesmanlaan 121, 1066CX Amsterdam, The Netherlands. Phone: 31.20.5121942; Fax: 31.20.5211944; E-mail: a.sonnenberg@nki.nl.

Norman Sachs's present address is: Hubrecht Institute KNAW and University Medical Center, Utrecht, The Netherlands.
1. Kriz W, Gretz N, Lemley KV. Progression of glomerular diseases: is the podocyte the culprit? Kidney Int. 1998;54(3):687-697.

2. Sachs $\mathrm{N}$, et al. Kidney failure in mice lacking the tetraspanin CD151. J Cell Biol. 2006;175(1):33-39.

3. Kanasaki $\mathrm{K}$, et al. Integrin beta1-mediated matrix assembly and signaling are critical for the normal development and function of the kidney glomerulus. Dev Biol. 2008;313(2):584-593.

4. Pozzi A, et al. Beta1 integrin expression by podocytes is required to maintain glomerular structural integrity. Dev Biol. 2008;316(2):288-301.

5. Dai C, et al. Essential role of integrin-linked kinase in podocyte biology: Bridging the integrin and slit diaphragm signaling. J Am Soc Nephrol. 2006; 17(8):2164-2175.

6. El Aouni C, et al. Podocyte-specific deletion of integrin-linked kinase results in severe glomerular basement membrane alterations and progressive glomerulosclerosis. J Am Soc Nephrol. 2006; 17(5):1334-1344

7. Kaplan JM, et al. Mutations in ACTN4, encoding alpha-actinin- 4 , cause familial focal segmental glomerulosclerosis. Nat Genet. 2000;24(3):251-256.

8. Zenker $\mathrm{M}$, et al. Human laminin beta2 deficiency causes congenital nephrosis with mesangial sclerosis and distinct eye abnormalities. Hum Mol Genet. 2004; 13(21):2625-2632.

9. Jarad G, Pippin JW, Shankland SJ, Kreidberg JA, Miner JH. Dystroglycan does not contribute significantly to kidney development or function, in health or after injury. Am J Physiol Renal Physiol. 2011; 300(3):F811-F820.

10. Baleato RM, Guthrie PL, Gubler MC, Ashman LK, Roselli S. Deletion of CD151 results in a strain- dependent glomerular disease due to severe alterations of the glomerular basement membrane. Am J Pathol. 2008;173(4):927-937.

11. Takeda Y, et al. Deletion of tetraspanin Cd151 results in decreased pathologic angiogenesis in vivo and in vitro. Blood. 2007;109(4):1524-1532.

12. Karamatic Crew V, et al. CD151, the first member of the tetraspanin (TM4) superfamily detected on erythrocytes, is essential for the correct assembly of human basement membranes in kidney and skin. Blood. 2004;104(8):2217-2223.

13. Yauch RL, Berditchevski F, Harler MB, Reichner J, Hemler ME. Highly stoichiometric, stable, and specific association of integrin alpha3beta 1 with CD151 provides a major link to phosphatidylinositol 4-kinase, and may regulate cell migration. $\mathrm{Mol}$ Biol Cell. 1998;9(10):2751-2765.

14. Sterk LM, Geuijen CA, Oomen LC, Calafat J, Janssen $\mathrm{H}$, Sonnenberg A. The tetraspan molecule CD151, a novel constituent of hemidesmosomes, associates with the integrin alpha6beta 4 and may regulate the spatial organization of hemidesmosomes. J Cell Biol. 2000;149(4):969-982.

15. Kreidberg JA, et al. Alpha 3 beta 1 integrin has a crucial role in kidney and lung organogenesis. Development. 1996;122(11):3537-3547.

16. Nishiuchi R, et al. Ligand-binding specificities of laminin-binding integrins: a comprehensive survey of laminin-integrin interactions using recombinant alpha3beta1, alpha6beta1, alpha7beta1 and alpha6beta4 integrins. Matrix Biol. 2006;25(3):189-197.

17. Van der Neut R, Krimpenfort P, Calafat J, Niessen $\mathrm{CM}$, Sonnenberg A. Epithelial detachment due to absence of hemidesmosomes in integrin beta 4 null mice. Nat Genet. 1996;13(3):366-369.
18. Nishiuchi R, et al. Potentiation of the ligand-binding activity of integrin alpha3beta 1 via association with tetraspanin CD151. Proc Natl Acad Sci U S A. 2005;102(6):1939-1944.

19. Sincock PM, Mayrhofer G, Ashman LK. Localization of the transmembrane 4 superfamily (TM4SF) member PETA-3 (CD151) in normal human tissues: comparison with CD9, CD63, and alpha5beta 1 integrin. J Histochem Cytochem. 1997;45(4):515-525.

20. Moeller MJ, Sanden SK, Soofi A, Wiggins RC, Holzman LB. Podocyte-specific expression of cre recombinase in transgenic mice. Genesis. 2003;35(1):39-42.

21. Mundel P, Shankland SJ. Podocyte biology and response to injury. J Am Soc Nephrol. 2002; 13(12):3005-3015.

22. Kambham N, et al. Congenital focal segmental glomerulosclerosis associated with beta4 integrin mutation and epidermolysis bullosa. Am J Kidney Dis. 2000;36(1):190-196.

23. Takemoto $M$, et al. A new method for large scale isolation of kidney glomeruli from mice. Am J Pathol. 2002;161(3):799-805.

24. Kazarov AR, Yang X, Stipp CS, Sehgal B, Hemler ME. An extracellular site on tetraspanin CD151 determines alpha 3 and alpha 6 integrin-dependent cellular morphology. J Cell Biol. 2002;158(7):1299-1309.

25. Gailit J, Ruoslahti E. Regulation of the fibronectin receptor affinity by divalent cations. JBiol Chem. 1988; 263(26):12927-12932.

26. Wright MD, et al. Characterization of mice lacking the tetraspanin superfamily member CD151. Mol Cell Biol. 2004;24(13):5978-5988.

27. Dworkin LD, Benstein JA, Parker M, Tolbert E, Feiner HD. Calcium antagonists and converting enzyme inhibitors reduce renal injury by different 
mechanisms. Kidney Int. 1993;43(4):808-814.

28. Harris DC, Hammond WS, Burke TJ, Schrier RW. Verapamil protects against progression of experimental chronic renal failure. Kidney Int. 1987; 31(1):41-46.

29. Chattopadhyay N, Wang Z, Ashman LK, BradyKalnay SM, Kreidberg JA. alpha3beta1 integrinCD151, a component of the cadherin-catenin complex, regulates PTPmu expression and cell-cell adhesion. J Cell Biol. 2003;163(6):1351-1362.

30. Yanez-Mo M, et al. Regulation of endothelial cell motility by complexes of tetraspan molecules CD81/ TAPA- 1 and CD151/PETA-3 with alpha3 beta 1 integrin localized at endothelial lateral junctions. $J$ Cell Biol. 1998;141(3):791-804.

31. Yanez-Mo M, Tejedor R, Rousselle P, Madrid F. Tetraspanins in intercellular adhesion of polarized epithelial cells: spatial and functional relationship to integrins and cadherins. J Cell Sci. 2001; 114(pt 3):577-587.

32. Sterk LM, Geuijen CA, van den Berg JG, Claessen N, Weening JJ, Sonnenberg A. Association of the tetraspanin $\mathrm{CD} 151$ with the laminin-binding integrins alpha3beta1, alpha6beta1, alpha6beta4 and alpha7 beta1 in cells in culture and in vivo. J Cell Sci. 2002; 115(pt 6):1161-1173.
33. Yanez-Mo M, Barreiro O, Gordon-Alonso M, SalaValdes M, Sanchez-Madrid F. Tetraspanin-enriched microdomains: a functional unit in cell plasma membranes. Trends Cell Biol. 2009;19(9):434-446.

34. Ashman LK, Fitter S, Sincock PM, Nguyen L, Cambareri A. CD151 (PETA-3) Workshop Summary Report. Kishimoto T, ed. New York, New York, USA: Garland Publishing; 1997:681-683.

35. Hemler ME, et al. Glycoproteins of 210,000 and 130,000 m.w. on activated T cells: cell distribution and antigenic relation to components on resting cells and T cell lines. J Immunol. 1984;132(6):3011-3018.

36. de Melker AA, et al. The A and B variants of the alpha 3 integrin subunit: tissue distribution and functional characterization. Lab Invest. 1997; 76(4):547-563.

37. Sonnenberg A, Janssen H, Hogervorst F, Calafat J, Hilgers J. A complex of platelet glycoproteins Ic and IIa identified by a rat monoclonal antibody. J Biol Chem. 1987;262(21):10376-10383.

38. Von Ballestrem CG, Uniyal S, McCormick JI, Chau T, Singh B, Chan BM. VLA-beta 1 integrin subunitspecific monoclonal antibodies MB1.1 and MB1.2: binding to epitopes not dependent on thymocyte development or regulated by phorbol ester and divalent cations. Hybridoma. 1996;15(2):125-132.
39. Baldwin HS, et al. Platelet endothelial cell adhesion molecule-1 (PECAM-1/CD31): alternatively spliced, functionally distinct isoforms expressed during mammalian cardiovascular development. Development. 1994;120(9):2539-2553.

40. Muzumdar MD, Tasic B, Miyamichi K, Li L, Luo L. A global double-fluorescent Cre reporter mouse. Genesis. 2007;45(9):593-605

41. Raymond K, Kreft M, Janssen H, Calafat J, Sonnenberg A. Keratinocytes display normal proliferation, survival and differentiation in conditional beta4-integrin knockout mice. J Cell Sci. 2005; 118(pt 5):1045-1060.

42. Anton M, Graham FL. Site-specific recombination mediated by an adenovirus vector expressing the Cre recombinase protein: a molecular switch for control of gene expression. J Virol. 1995;69(8):4600-4606.

43. Delwel GO, Hogervorst F, Kuikman I, Paulsson M, Timpl R, Sonnenberg A. Expression and function of the cytoplasmic variants of the integrin alpha 6 subunit in transfected K562 cells. Activation-dependent adhesion and interaction with isoforms of laminin. J Biol Chem. 1993;268(34):25865-25875.

44. Boettiger D. Quantitative measurements of integrin-mediated adhesion to extracellular matrix. Methods Enzymol. 2007;426:1-25. 